\section{OP36 DECISIONS ABOUT SMOKING IN PATIENTS SCREENED WITH THE EARLYCDT-LUNG TEST FOR THE EARLY DETECTION OF LUNG CANCER: A QUALITATIVE STUDY}

${ }^{1} \mathrm{~B}$ Young ${ }^{*},{ }^{1} \mathrm{~K}$ Vedhara, ${ }^{1} \mathrm{D}$ Kendrick, ${ }^{2} \mathrm{~J}$ Robertson, ${ }^{3} \mathrm{R}$ das Nair. ${ }^{1}$ Division of Primary Care, University of Nottingham, Nottingham, UK; ${ }^{2}$ Division of Medical Sciences and Graduate Entry Medicine, University of Nottingham, Nottingham, UK; ${ }^{3}$ Division of Psychiatry and Applied Psychology, University of Nottingham, Nottingham, UK

\subsection{6/jech-2017-SSMAbstracts.36}

Background Routine screening for lung cancer in high risk groups (characterised by age and smoking history) is recommended in the USA and may be implemented elsewhere. It is unclear whether being screened for lung cancer promotes smoking cessation or conversely provides false reassurance and a 'license to smoke'. This study aimed to understand how experiences of lung cancer screening influence individual decision making about smoking.

Methods Thirty one people in Scotland, aged 51-74, took part in semi-structured interviews. They had been screened with the EarlyCDT-Lung Test (13 positive result; 18 negative) as part of the Early Cancer Detection Test-Lung Cancer Scotland (ECLS) Study and were long-term smokers when screened. Verbatim transcripts were analysed using thematic analysis.

Results Interpretations of test results was a key theme, but were often inaccurate, for example a negative result interpreted as an 'all-clear' from lung cancer and a positive result as meaning lung cancer will definitely develop. There was no clear pattern in decisions made about smoking in response to positive or negative test results. Emotional response to those interpretations was an overarching theme in decisions about smoking. Emotions included fear, shock, upset, worry, anxiety, guilt, relief, reassurance and indifference. Other themes included changes in perceived risk of smoking-related disease, a feeling that now is the time to stop smoking, interpersonal family influences and avoidance of thoughts about smoking. Of those who had stopped smoking, some cited screening experiences as the sole reason and some cited screening along with other coinciding factors. Cues to change were experienced at different stages of the screening process and not always immediately following a test result. Some participants indicated they underwent screening in order to try and stop smoking. Others expressed little or no desire to stop. In general, lung cancer screening was experienced as a unique opportunity, which sometimes prompted successful or unsuccessful attempts to stop smoking.

Conclusion Lung cancer screening can be a 'teachable moment' for smoking behaviour change. Emotional responses to test results, which can be misinterpreted, were an important theme but behavioural responses varied according to the individual. Findings should be considered within the context of a group of predominantly life-long smokers undergoing a novel blood screening test, who might already have increased motivation to stop smoking. Lung cancer screening presents an opportunity to engage high risk smokers in cessation support but our findings suggest such support may need to be available flexibly to be most effective.

In collaboration with the ECLS study team

\section{Ethnicity and children}

\section{OP37 ETHNIC VARIATION IN RISK OF SUDDEN INFANT DEATH SYNDROME AND OTHER UNEXPLAINED INFANT DEATH IN ENGLAND AND WALES 2006-2012: NATIONAL BIRTH COHORT WITH ROUTINE EXPOSURE AND OUTCOME DATA}

${ }^{1}$ ME Kroll ${ }^{*},{ }^{1} \mathrm{MA}$ Quigley, ${ }^{2} \mathrm{~N}$ Dattani, ${ }^{1} \mathrm{~J} \mathrm{Hollowell} .{ }^{1}$ Policy Research Unit in Maternal Health and Care, National Perinatal Epidemiology Unit, University of Oxford, Oxford, UK; ${ }^{2}$ School of Health Sciences, City, University of London, London, UK

\subsection{6/jech-2017-SSMAbstracts.37}

Background About $8 \%$ of deaths under age 1 year in England and Wales are unexplained, the cause of death being recorded as sudden infant death syndrome (SIDS, 60\%) or unascertained (40\%). Typically, unexplained infant death (UID) occurs during sleep, perhaps triggered by unsafe sleep environments (prone/side position, soft bedding etc.). Other risk factors include preterm birth, which may increase vulnerability. Incidence of both preterm birth and (in an analysis of data from 2005) SIDS has been relatively high for Black Caribbean babies. We aimed to evaluate recent ethnic variation in UID, test the hypothesis that it might be mediated by preterm birth, and assess several other potential explanatory factors.

Methods For singleton live births at gestation $22+$ weeks in England and Wales during 2006-2012, the Office of National Statistics provided a file linking birth and death registrations with other routine data. Variables included ethnicity (11 groups) and six covariates: gestational age, area deprivation (IMD quintile), gender, mother's age at delivery, mother's country of birth (UK-born or not), parental status at birth registration (within marriage, jointly registered by both parents at same address, joint registration with different addresses, sole registration). Using Stata, we fitted a sequence of logistic regression models for UID, starting with ethnicity and adding factors in the order in which they reduced the likelihood ratio test statistic for ethnic variation.

Results There were 4.6 million births and 1559 UID events (0.34 per 1000). Risk varied by ethnicity $(\mathrm{p}<0.001)$, being low for Black African, White non-British, and all South Asian groups, but high for Black Caribbean and two Mixed Black groups (African-White and Caribbean-White). Unadjusted odds ratios relative to White British ranged from 0.38 (95\% CI 0.24-0.60) for Indian to $1.73(1.21-2.47)$ for Black Caribbean. All covariates were associated with UID risk. When jointly adjusted for two factors (parental registration status and mother's country of birth), the ethnic variation was not statistically significant $(p=0.6)$. Other factors, including gestational age, made very little difference. Results were robust to sensitivity analysis for potential sparse-data bias.

Conclusion This large population-based study found substantial ethnic disparity in risk of UID, which was not attributable to preterm birth, maternal age or area deprivation. Parental registration status and mother's country of birth jointly accounted for the variation, suggesting mediation by cultural factors. Better understanding of infant care practices in low-risk ethnic groups might lead to more effective prevention of UID in the general population. 